to their questions through the Internet or from healthcare professionals. Three different description categories emerged: Specific competence, Constructive dialogue, and Applicability. The informants' perceived Specific competence when the nurses were knowledgeable, the call was complementary to previously received information and when the informants had greater knowledge after the contact with RD. They perceived that it was a Constructive dialogue when they got someone to discuss with, a "sounding board", and perceived emotional support, felt reassured and were satisfied with the answer. The informants perceived Applicability because RD was available and they could make different choices according to their own desire; before (how and when they would contact RD), during (what to tell and what question they would ask) and after (how and what they would do after the contact with RD).

Conclusions: People calling RD perceived that the telephone call with the nurses meant meeting specific competence, gaining constructive dialogue and that the helpline was applicable. This knowledge ad to a fuller understanding of factors that from a caller's perspective, are important when calling a helpline with specially trained nurses on rheumatic diseases.

Disclosure of Interest: None declared

DOI: 10.1136/annrheumdis-2017-eular.2466

\section{AB1239-HPR FATIGUE AT DIAGNOSIS OF INFLAMMATORY JOINT DISEASES - A PREDICTOR OF FATIGUE DURING THE COURSE OF DISEASE DESPITE OF LOW DISEASE ACTIVITY}

S.H. Jakobsen, A. Emamifar, I.M.J. Hansen. Rheumatology, OUH, Svendborg Hospital, Svendborg, Denmark

Background: Fatigue is a common symptom in patients suffering from inflammatory rheumatic diseases. Several patients still present with fatigue, although they are well treated with anti TNF-therapy (1).

Objectives: To investigate disease-related aspects of fatigue in patients with inflammatory rheumatic diseases using the Functional Assessment of Chronic Illness Therapy-Fatigue (FACIT-F) with the aim later to develop methods to improve the patients quality of life in a more specific way.

Methods: All patients with inflammatory diseases including Rheumatoid Arthritis (38) and Spondyloarthropathy (13) and Psoriatic arthritis (2) treated with Intravenous biologic from 15.10 until 31.12.16 were invited to fill out the FACIT-F questionnaire during intravenous (IV) infusion of the drugs in the rheumatology outpatient clinic. Furthermore hemoglobin and disease activity score were extracted from patients electronic records.

Results: Of 72 patients, 53 patients completed the questionnaire. 5 patients did not want to participate. In 11 patients treatment was discontinued during the study and 3 patients were not able to answer the questions.

Patients with a fatigue score of $<30$ had few problems with any of the subgroups within the FACIT-F questionnaire (A-E), whereas more than $30 \%$ of patients with a fatigue score of $\geq 30$ had challenges in one of the FACIT-F subgroups (somewhat, quite a bit and very much) A. Physical well-being: lack of energy and troubles with meeting the needs of their family because of their physical condition. B. Social/family well-being: patients were not feeling close to their friends, not getting enough emotional support from their family and not satisfied with their sex life. C. Emotional well-being: patients worry if their conditions might get worse. D. Functional well-being: patients feel they are partly unable to work, not satisfied with their performance at work and they have sleeping and quality of life problems. E. Additional concerns: patients had problems with fatigue, weakness, tiredness, starting and finishing things because of tiredness, not having energy, not being able to do usual activities, frustration by being too tired to do the things they want to do as well as they want to limit social activities because of tiredness and they need to sleep during the day. Furthermore, there was a moderate correlation between fatigue at diagnosis and fatigue at time of data extraction $(r=0.53)$. The fatigue was not correlated with anemia or high disease activity.

Conclusions: Our results demonstrated that patients with a fatigue score of $>30$ had different challenges mentioned in the fatigue questionnaire. In addition, patients who experience fatigue at time of diagnosis, they often remain fatigue, provoking the thought that fatigue is not correlated with inflammatory joint disease. Maybe more explorative questions about fatigue at the consultation could be a part of improving the patients'quality of life.

References:

[1] Druce KL, et al. Most patients who reach disease remission following antiTNF therapy continue to report fatigue: results from the British Society for Rheumatology Biologics Register for Rheumatoid Arthritis. Rheumatology (Oxford). 2016;55:1786-1790.

[2] Webster K, Cella D, Yost K. The Functional Assessment of Chronic IIIness Therapy (FACIT) Measurement System: properties, applications, and interpretation. Health Qual Life Outcomes 2003;1:79.

Disclosure of Interest: None declared

DOI: 10.1136/annrheumdis-2017-eular.1885

\section{AB1240-HPR PATIENTS' DOGMA, NUMBER OF SWOLLEN JOINTS AND PHYSICIANS' AND PATIENTS' AGE PREDICT NON-ADHERENCE TO MEDICINES AND NON-PHARMACOLOGICAL INTERVENTIONS IN RHEUMATOID ARTHRITIS - A MIXED METHODS STUDY}

V. Ritschl ${ }^{1}$, A. Lackner ${ }^{2}$, C. Boström ${ }^{3}$, M. Kundi ${ }^{4}$, P. Studenic ${ }^{5}$, J. Smolen ${ }^{5}$, T. Stamm ${ }^{1} .{ }^{1}$ Institute of Outcomes Research, Center for Medical Statistics, Informatics, and Intelligent Systems, Medical University of Vienna, Vienna; ${ }^{2}$ Department of Rheumatology, Medical University of Graz, Graz, Austria; ${ }^{3}$ Department of Neurobiology, Karolinska Institute, Huddinge, Sweden; ${ }^{4}$ Institute of Environmental Health, Center for Public Health; ${ }^{5}$ Division of Rheumatology, Department of Medicine 3, Medical University of Vienna, Vienna, Austria

Background: In rheumatoid arthritis (RA), up to $80 \%$ of patients were found to be non-adherent to prescribed medication and non-pharmacological recommendations. These patients do not achieve an optimal clinical outcome.

Objectives: In the present study, we therefore explored predictors that may lead to non-adherence to both medicines and/or non-pharmacological recommendations. Methods: In a mixed methods study, retrospective observational data from patients meeting the ACR/EULAR criteria for RA who were non-attenders/missed the routine check up visits for at least 9 months to the rheumatology clinic and had had an initial DMARD therapy were queried of the databases of two rheumatology centers in Austria (Graz, Vienna). Subsequently, we invited all patients to take part in a qualitative semi-structured interview study with a meaning condensation data analysis. In the interviews, patients were assigned to the subgroups "adherent" (e.g. having regular rheumatology visits in another clinic) or "non-adherent" (e.g. having stopped taking the prescribed medication). Possible predictors derived from the qualitative analysis and the retrospective observational data were then tested in a logistic regression model.

Results: In total, data of 459 patients (346 [75.4\%] females; mean age 63.0 [SD \pm 14.8]) were extracted out of the databases. 131 patients (109 [83.2\%] females; mean age 64.8 [SD \pm 14.1$]$ ) participated in the qualitative interviews. In addition to already known themes, new topics arose from the analysis: (i) patient's dogma inhibited adherent behavior, in that patients felt that pain was an important part of life and attributed to having had a high manual workload during life of which patients were proud; (ii) patients had less trust in physicians when they were seeking support from other physicians, because they appeared to be "young or unexperienced"; (iii) Some patients did not feel properly understood if physicians only prescribed medication without giving advice on non-pharmacological aspects of treatment.

Two clinical variables were found to be predictors for non-adherent behavior (table 1): swollen joint count (patients with higher numbers of swollen joints were less adherent) and age (younger patients were less adherent).

Table 1. Logistic regression models. Odds ratios of relevant factors for non-adherent behavior in RA: results of the logistic regression analysis

\begin{tabular}{lccc}
\hline Regression Model & Odds Ratio & $\mathrm{Cl} 95 \%$ & Significance at $0.05(\mathrm{p})$ \\
\hline $\begin{array}{l}\text { Age at the last visit } \\
\begin{array}{l}\text { Swollen Joint Count using a 32 joint } \\
\text { count form }\end{array}\end{array}$ & 1.033 & 1.005 to 1.063 & 0.022 \\
\hline
\end{tabular}

Conclusions: In order to achieve a good clinical outcome, it is important to provide evidence based treatment recommendations, but also to ensure adherence to these. The predictors found in our study could be used to enhance patient adherence and therefore improve clinical outcome.

Acknowledgements: This project was partially funded by AbbVie Inc.

Disclosure of Interest: None declared

DOI: 10.1136/annrheumdis-2017-eular.5227

\section{AB1241-HPR EVALUATION OF PATIENT COMPLIANCE WITH LONG TERM PRESCRIBED RHEUMATIC MEDICATION AT LOCAL LONDON HOSPITAL RHEUMATOLOGY UNIT}

V. Sohdi, C.B. Colaço, M. Hoffman, J. Kirk on behalf of Central Middlesex Hospital Rheumatology (Patient) Support Group. LNWHT, London, United Kingdom

Background: Non-Compliance with Long term medication is reported as high $60 \%$ (1). Health belief model suggests four elements contribute to this problem. The Beliefs About Medicines Questionnaire (BMQ) is a tool for evaluating people's beliefs about medicines (1).

Objectives: A Service Evaluation of compliance with prescribed medication for Long Term Rheumatologic Conditions at Central Middlesex Hospital. This was conducted to assess any possible relationship between compliance with medicines and beliefs or concerns or patient's ethnicity.

Methods: The design was a voluntary self-reported, cross-sectional paper based questionnaire survey of people with Rheumatic Conditions. Twelve questions were grouped within three categories (healthcare utilisation, necessity beliefs and concern beliefs) to capture compliance behaviour for later analysis and comparison.

The Beliefs about Medicines Questionnaire was adapted from ref (1) to distinguish patients beliefs of 'necessity' or 'concerns'. In line with principles of PPP, the questionnaire was discussed prior to the audit with a sample focus group of 5 